\title{
DECONVOLUTION TECHNIQUES FOR LINEAR SYSTEMS
}

\author{
By Hon-Yim Ko and Ronadi F. Scott
}

\begin{abstract}
Superposition for linear systems is the process in which the input is convolved with the transfer function of the system, whereby the output is obtained. Deconvolution is, therefore, the reverse process in which an unknown input (or the transfer function) is calculated from the measured output and a known transfer function (or input). Several methods of deconvolution are developed and the advantages and disadvantages of each are discussed. Examples are given to demonstrate the applicability of the methods.
\end{abstract}

\section{INTRODUCTION}

The most important property of a linear system is that the principle of superposition is applicable. Mathematically, this principle can be expressed as (Fodor, 1965)

$$
f(t)=\int_{0}^{t} l(t-\tau) w(\tau) d \tau
$$

where

$l(t)=$ input to the system

$f(t)=$ output from the system

and $w(t)=$ system function or transfer function.

Equation (1) is commonly known as the superposition, convolution, or Duhamel integral. The system function, $w(t)$, is the response, or output, of the system when perturbed by an impulse input. It is also equivalent to the time-derivative of the response of the system due to a step loading. The system function can therefore be determined by subjecting the system to either an impulse or a step loading.

However, in real systems, such loadings may be difficult to apply, and consequently it is desirable to determine $w(t)$ by measuring the output $f(t)$ due to any input $l(t)$ to the system. For this purpose, equation (1) can be treated as an integral equation for the unknown $w(t)$ with a kernel $l(t)$. Alternately, equation (1) can also be written as

$$
f(t)=\int_{0}^{t} w(t-\tau) l(\tau) d \tau
$$

which is then an integral equation for $l(t)$. Hence, the roles of $w(t)$ and $l(t)$ in these equations are completely interchangeable, and if $w(t)$ can be determined from equation (1), so can $l(t)$ from equation (2). The utilization of equation (2) is useful in situations where it is required to calculate the loading $l(t)$ which gives rise to a measured output from a system with a known system function $w(t)$. The solution of these convolution-type integral equations is called "deconvolution".

Examples where this type of integral equation is applicable can be found in 
engineering (Greenwood, 1965), physics (Courant and Hilbert, 1953) and biology (Beck and Rescigno, 1964). In the following a few examples will be mentioned and methods for solving these equations will be developed.

\section{The Transform Method}

Equation (1) or (2) belongs to a class of linear integral equations of the Volterra type of the first kind (Triconi, 1957). One way of solving this type of equation is by transform techniques. First, (1) or (2) can be written in the form

$$
f(t)=l(t) * w(t)=w(t) * l(t),
$$

where * represents the convolution. Applying the Laplace transform to (3), the following result is obtained:

$$
F(s)=L(s) W(s)
$$

where $F(s), L(s)$ and $W(s)$ are the Laplace transforms of $f(t), l(t)$ and $w(t)$ respectively. Depending on whether $l(t)$ or $w(t)$ is the desired solution, (4) can be written as

$$
L(s)=\frac{F(s)}{W(s)}
$$

or

$$
W(s)=\frac{F(s)}{L(s)} .
$$

Applying the inverse transform to these equations, the required solutions are expressed as

$$
\begin{aligned}
l(t)=\mathfrak{L}^{-1}[L(s)] & =\mathfrak{L}^{-1}\left[\frac{F(s)}{W(s)}\right]=\mathfrak{L}^{-1}[F(s)] * \mathfrak{L}^{-1}\left[\frac{1}{W(s)}\right] \\
& =f(t) * \mathfrak{L}^{-1}\left[\frac{1}{W(s)}\right]
\end{aligned}
$$

or, similarly,

$$
w(t)=f(t) * \mathfrak{L}^{-1}\left[\frac{1}{L(s)}\right]
$$

In general, for a given function $w(t)$ for which $W(s)$ exists, $\mathfrak{L}^{-1}[1 / W(s)]$ does not exist or is difficult to evaluate. The same is true if $w(t)$ is substituted by $l(t)$. Therefore, this method of directly applying the Laplace transform to (1) or (2) runs into difficulty. 
The Volterra integral equation of the first kind, (1) or (2), can be reduced to one of the second kind by differentiating the equation. (In the following, only equation (2) will be discussed, although the results are equally applicable to (1) by interchanging $w(t)$ and $l(t))$. Differentiating (2) with respect to time, $t$, the following is obtained:

$$
f^{\prime}(t)=\int_{0}^{t} w^{\prime}(t-\tau) l(\tau) d \tau+w(0) l(t)
$$

or

$$
h(t)=\int_{0}^{t} v(t-\tau) l(\tau) d \tau+l(t)
$$

where

$$
h(t)=\frac{f^{\prime}(t)}{w(0)}
$$

and

$$
v(t)=\frac{w^{\prime}(t)}{w(0)}
$$

Equation (7), a Volterra integral equation of the second type, can be transformed to

$$
H(s)=V(s) L(s)+L(s)
$$

from which

$$
L(s)=\frac{H(s)}{1+V(s)}=H(s)-\frac{V(s)}{1+V(s)} H(s)
$$

If $V(s)$ exists, then $\mathfrak{L}^{-1}[V(s) /(1+V(s))]$ also exists (Fodor, 1965). Therefore, (8) can be inverse-transformed to

$$
\begin{aligned}
l(t) & =h(t)-\left[\mathfrak{e}^{-1}\left(\frac{V(s)}{1+V(s)}\right)\right] * h(t) \\
& =\frac{f^{\prime}(t)}{w(0)}-\mathfrak{L}^{-1}\left[\frac{V(s)}{1+V(s)}\right] *\left[\frac{f^{\prime}(t)}{w(0)}\right] .
\end{aligned}
$$

This approach is satisfactory if $w(0)$ is some finite number If it is not, then the following method can be used. 
Let

$$
\begin{aligned}
u(t) & =\int_{0}^{t} l(\tau) d \tau . \\
U(s) & =\frac{1}{s} L(s) .
\end{aligned}
$$

Substitute into equation (5a) to obtain

$$
U(s)=\frac{F(s)}{s W(s)}=M(s) F(s)
$$

where

$$
M(s)=\frac{1}{s W(s)}
$$

Obtain the inverse transform

$$
\begin{aligned}
u(t) & =m(t) * f(t) \\
& =\int_{0}^{t} m(t-\tau) f(\tau) d \tau \\
& =\int_{0}^{t} m(\tau) f(t-\tau) d \tau .
\end{aligned}
$$

If $f(t)$ is differentiable, then

$$
u^{\prime}(t)=l(t)=f(0) m(t)+\int_{0}^{t} m(\tau) f^{\prime}(t-\tau) d \tau
$$

where

$$
m(t)=\mathfrak{L}^{-1}[M(s)]=\mathfrak{L}^{-1}\left[\frac{1}{s W(s)}\right] .
$$

It should be mentioned that instead of using Laplace transforms, other linear transformations such as Fourier transforms can also be used. However, the same difficulties will appear in connection with obtaining the inverse transforms. An example is next shown in which the method employed in arriving at equation (10) is used to determine the loading function $l(t)$, given the system and the response functions.

Example 1. In this example, the problem is the one-dimensional diffusion equation,

$$
\frac{\partial^{2} \theta}{\partial x^{2}}=\frac{1}{\alpha} \frac{\partial \theta}{\partial t}
$$


The particular application may be heat diffusion (Carslaw and Jaeger, 1959) where $\theta$ is the temperature and $\alpha$ is the thermal diffusivity, or it may be consolidation in soil mechanics (Scott, 1963), in which case $\theta$ is the excess pore water pressure, and $\alpha$ is the coefficient of consolidation. The latter case will be examined.

Equation (11) can be nondimensionalized to

$$
\frac{\partial^{2} \theta}{\partial x^{2}}=\frac{\partial \theta}{\partial t}
$$

where $\theta, x$ and $t$ are now the dimensionless pressure, dimensionless distance and dimensionless time respectively.

The problem selected is one in which the surface of a semi-infinite medium is subjected to a ramp loading in which the pressure increases linearly with time up to a certain time and then is held constant. The average consolidation or the settlement of the surface of the medium, $\bar{U}(t)$, is measured as a function of time. The deconvolution method will be applied to the situation where the response $f(t)$ $(=U(t))$ and the system function $w(t)$ are known and $l(t)$ is treated as the unknown. Since the input function is known, it can be used for comparison with the input function calculated by the deconvolution process.

The average consolidation of the medium under a unit impulse load is given by

$$
w(t)=2 \sum_{n=0}^{\infty} \exp ^{-\pi^{2}(2 n+1) 2^{2} t / 4}
$$

which is the system function for this problem. The Laplace transform of $w(t)$ is

$$
W(s)=2 \sum_{n=0}^{\infty} \frac{1}{s+\frac{\pi^{2}}{4}(2 n+1)^{2}} .
$$

so that

$$
\begin{gathered}
M(s)=\frac{1}{s W(s)}=\frac{1}{2 s \sum_{n=0}^{\infty} \frac{1}{s+\frac{\pi^{2}}{4}(2 n+1)^{2}}} \\
=\frac{-\frac{1}{2 s}}{s+\frac{\pi^{2}}{4}+\frac{2 s}{s+\frac{9 \pi^{2}}{4}}+\frac{2 s}{s+\frac{25 \pi^{2}}{4}}+\cdots}
\end{gathered}
$$

The inverse transform of $M(s)$ is difficult to obtain if the infinite series is used. However, by retaining only the frist few terms in this series, a good approximation is obtained, as will be demonstrated. 
If only the first term is used, i.e.,

$$
M(s)=M^{(1)}(s)=\frac{s+\pi^{2} / 4}{2 s}=\frac{1}{2}+\frac{\pi^{2}}{8 s},
$$

the inverse Laplace transform is found to be

$$
m^{(1)}(t)=\frac{1}{2} \delta(t)+\frac{\pi^{2}}{8},
$$

where $\delta(t)$ is the Dirac or delta function.

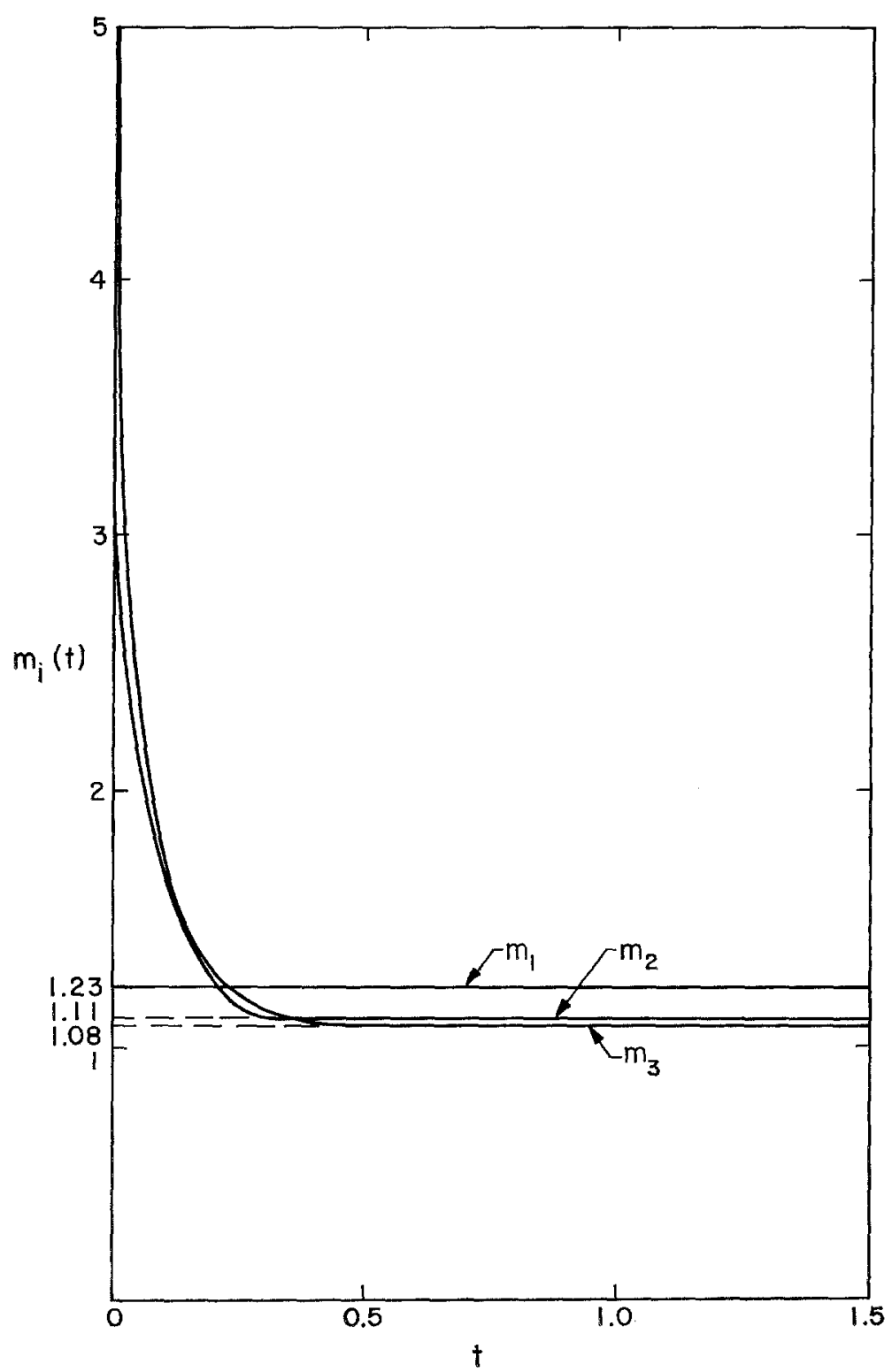

FIG. 1. Inverse transforms of the successive approximations of $m(t)$. 
If the first two terms in the series are retained, i.e.,

$$
M(s)=M^{(2)}(s)=\frac{1}{\frac{2 s}{s+\frac{\pi^{2}}{4}}+\frac{2 s}{s+\frac{9 \pi^{2}}{4}}}=\frac{1}{4}+\frac{9 \pi^{2}}{80 s}+\frac{\pi^{2}}{5\left(s+\frac{5 \pi^{2}}{4}\right)},
$$

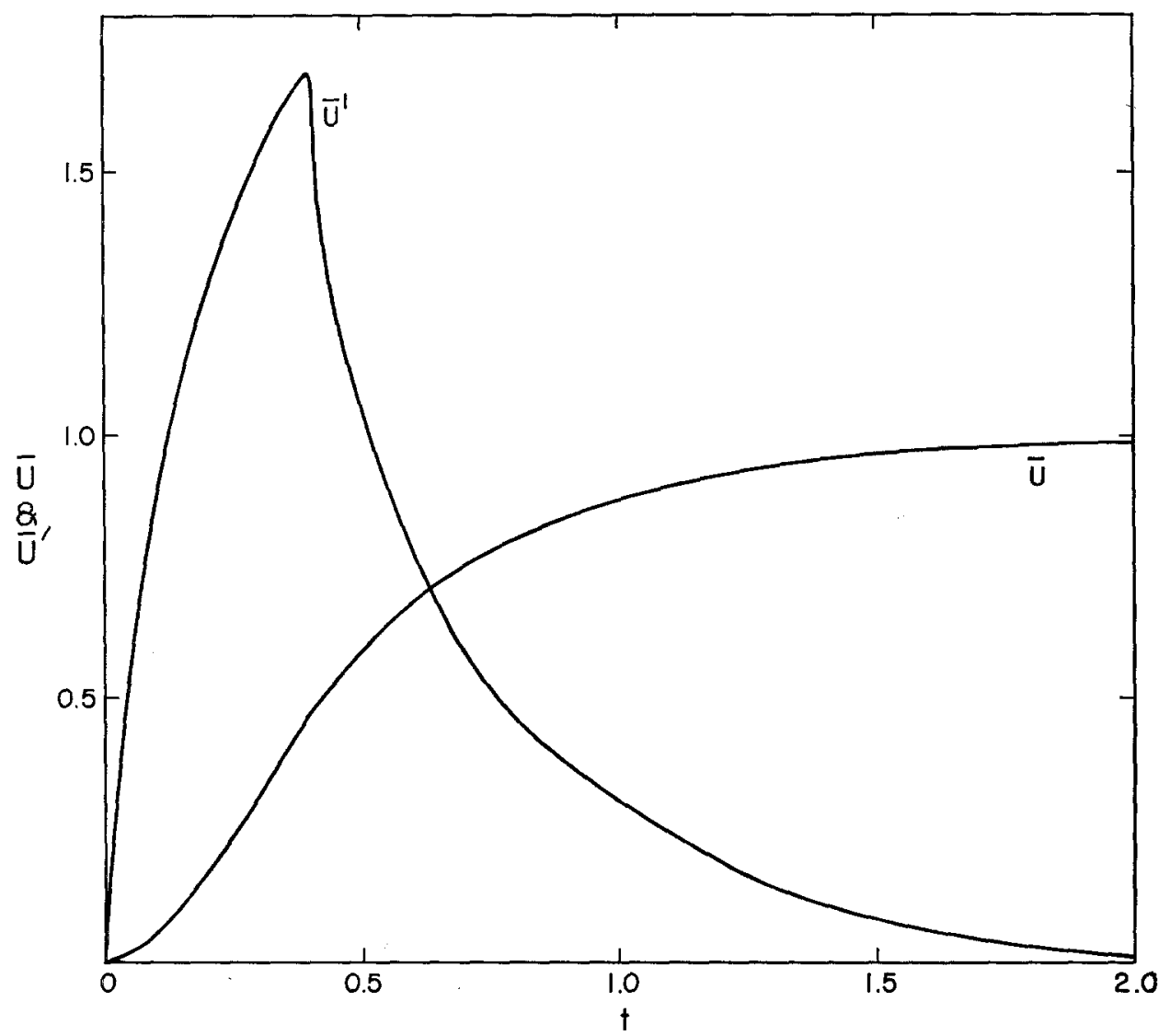

Fig. 2. System output for the consolidation problem, $\bar{U}(t)$ and $\bar{U}^{\prime}(t)$.

then

$$
m^{(2)}(t)=\frac{1}{4} \delta(t)+\frac{9 \pi^{2}}{80}+\frac{\pi^{2}}{5} e^{-5 \pi^{2} t / 4}
$$

If $n$ terms are included in the series, then it will be seen that $m^{n}(t)$ will consist of the following terms:

(1) $(1 / 2 n) \delta(t)$,

(2) a constant term which approaches unity as $n \rightarrow \infty$, and

(3) a series of exponential terms of the form $e^{-\alpha_{i} t}$, where $\alpha_{i}$ becomes much larger than unity as $n \rightarrow \infty$.

It is possible to plot these functions $m^{(i)}(t)$, except that due to the delta function, the values of these at $t=0$ are indeterminate. Figure 1 shows the first three ap- 
proximations to $m(t)$ which is the exact inversion of $M(s)$ in equation $(13)$. It can be seen that the second and the third approximations are already very close to each other.

The average consolidation $\bar{U}(t)$ due to a ramp loading is obtained by Schiffman) (1958) and shown in Figure 2. This is the response, $f(t)$, to be used in equation (10) and which will be assumed to have been measured. In Figure 2 is also shown $\bar{U}^{\prime}(t)$, which is $f^{\prime}(t)$.

The load, $l(t)$, is obtained by means of equation (10). A convolution is involved in the calculation and the following approximation is used for evaluating the convolution integral. If the convolution integral is as shown in equation (1), then it can

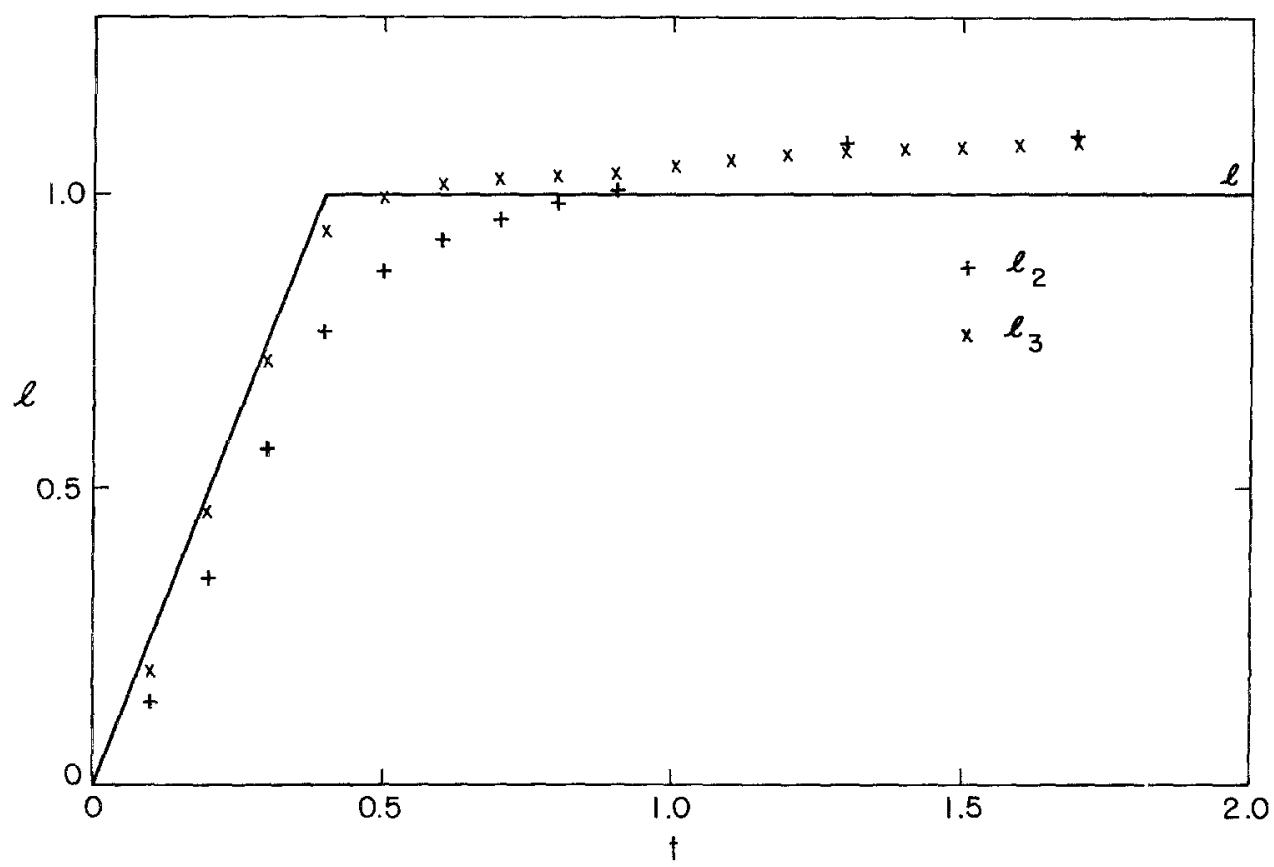

FIG. 3. Calculated system input for the consolidation problem by the transform technique, $l^{(i)}(t)$.

be evaluated numerically as

$$
f_{n}=f\left(t_{n}\right)=\Delta t \sum_{j=1}^{n} l_{j} w_{n-j+1}=\Delta t \sum_{j=1}^{n} l_{n-j+1} w_{j},
$$

where $l_{3}=$ average value of $l(t)$ between $t=(j-1) \Delta t$ and $j \Delta t$, and $w_{j}=$ average value of $v(t)$ between $t=(j-1) \Delta t$ and $j \Delta t$. By using the successive approximations $m^{(i)}(t)$ for $m(t)$ in equation (10), the loading functions $l^{(i)}(t)$ are shown in Figure 3 , where the actual correct ramp loading, $l(t)$, is also shown. Here, $\Delta t$ is equal to 0.1 . It can be seen that a very good approximation of $l(t)$ is obtained by using $m^{(3)}(t)$, bearing in mind that only three terms were retained in the infinite series in equation (13). If more terms in the series have been used, it is possible to achieve an even better reproduction of the loading function. Note also that a convergent approxi- 
mation is obtained by using more and more terms in the series. However, in another problem, this may not be true.

The method described and used in this example involves evaluating inverse transforms of arbitrary functions, which sometimes may be difficult. Although approximate inversion techniques have been developed in connection with viscoelastic stress analysis (Ter Haar, 1951; Schapery, 1962), they are too crude for use in connection with the deconvolution scheme. Since in most engineering problems very few of the functions in equation (1) can be expressed analytically, this deconvolution method must be applied in a numerical manner.

The numerical inversion of the Laplace transform has been studied (Bellman et $a l, 1966$ ), but the techniques used are complicated, involving quadrature with orthogonal polynomials. In the following section, a direct numerical technique is developed for the deconvolution, which has the additional advantage of providing a better physical understanding of the nature of the process.

\section{The Deconvolution by Matrix Methods}

If equation (16) is written in full, a set of $n$ linear algebraic equations is obtained

$$
\left.\begin{array}{l}
\frac{f_{1}}{\Delta t}=l_{1} w_{1} \\
\frac{f_{2}}{\Delta t}=l_{1} w_{2}+l_{2} w_{1} \\
\frac{f_{3}}{\Delta t}=l_{1} w_{3}+l_{2} w_{2}+l_{3} w_{1} \\
\ldots \ldots \ldots \ldots \ldots \ldots \ldots \ldots \ldots \ldots \\
\frac{f_{n}}{\Delta t}=l_{n} w_{1}+l_{n-1} w_{2}+\cdots \ldots+l_{2} w_{n-1}+l_{1} w_{n}
\end{array}\right\}
$$

If $l(t)$ is the unknown, (17) can be treated as $n$ equations containing $n$ unknowns, $l_{i}(i=1,--, n)$. In matrix notation, (17) can be written as

$$
[W]\{l\}=\frac{1}{\Delta t}\{f\}
$$

in which

$$
\begin{aligned}
& {[W]=\left[\begin{array}{lrrrrrr}
w_{1} & 0 & 0 & 0 & \ldots & \ldots & \ldots \\
w_{2} & w_{1} & 0 & 0 & \ldots & \ldots & \ldots \\
w_{3} & w_{2} & w_{1} & 0 & \ldots & \ldots & \ldots \\
\ldots & \ldots & \ldots & \ldots & \ldots & \ldots & \ldots \\
w_{n-1} & w_{n-2} & \ldots & \ldots & w_{2} & w_{1} & 0 \\
w_{n} & w_{n-1} & \cdots & w_{3} & w_{2} & w_{1}
\end{array}\right],} \\
& \{l\}=\left\{\begin{array}{c}
l_{1} \\
l_{2} \\
\vdots \\
l_{n}
\end{array}\right\},
\end{aligned}
$$


and

$$
\{f\}=\left\{\begin{array}{c}
f_{1} \\
f_{2} \\
\vdots \\
f_{n}
\end{array}\right\}
$$

The matrix $[W]$ has all its elements on the main diagonal equal to $w_{1}$, all its elements on successive diagonals below the main diagonal equal to $w_{2}, w_{3}, \cdots w_{n}$, and zeros above the main diagonal. Since $[W]^{-1}$ exists and equation (18) can there. fore be solved for $\{l\}$,

$$
\{l\}=\frac{1}{\Delta t}[W]^{-1}\{f\} \cdot=\frac{1}{\Delta t}[\bar{W}]\{f\} .
$$

It can be shown that $[\tilde{W}]$ has exactly the same form as $[W]$, i.e.,

$$
[\bar{W}]=\left[\begin{array}{lrrrrrrr}
\bar{w}_{1} & 0 & 0 & 0 & \ldots & \ldots & \ldots \\
\bar{w}_{2} & \bar{w}_{1} & 0 & 0 & \ldots & \ldots & \ldots \\
\bar{w}_{3} & \bar{w}_{2} & \bar{w}_{1} & 0 & \ldots & \ldots & \ldots \\
\cdots \ldots & \ldots & \ldots & \ldots & \ldots & \ldots & \ldots \\
\bar{w}_{n-1} & \bar{w}_{n-2} & \ldots & \ldots & \bar{w}_{2} & \bar{w}_{1} & 0 \\
\bar{w}_{n} & \bar{w}_{n-1} & \ldots & \bar{w}_{3} & \bar{w}_{2} & \bar{w}_{1}
\end{array}\right]
$$

and that $\bar{w}_{i}$ is related to $w_{i}$ as follows

$$
\left.\begin{array}{l}
\bar{w}_{1}=\frac{1}{w_{1}} \\
\bar{w}_{i}=-\sum_{j=1}^{i-1} \frac{w_{j+1}}{w_{1}} \bar{w}_{i-j}, i>1 .
\end{array}\right\}
$$

Hence, if $[W]$ is known, $[\vec{W}]$ can be easily calculated from equation (20) and then used in equation (19) to obtain $\{l\}$ required to produce an observed $\{f\}$. Since $[W]$ and $[\bar{W}]$ have the same form, then, by comparing equations (18) and (19), it is seen that the matrix multiplication in equation (19) represents just the numerical calculation of a convolution integral. Equation (19) is therefore equivalent to the convolution integral

$$
l(t)=\int_{0}^{t} \bar{w}(t-\tau) f(\tau) d \tau
$$

which is the solution of the original integral equation, equation (2). Since $[\bar{W}]$ is the inverse of $[W]$ and by its use the convolution integral can be solved, it is called the deconvolution matrix. Two examples are given to demonstrate the use of the deconvolution matrix. 


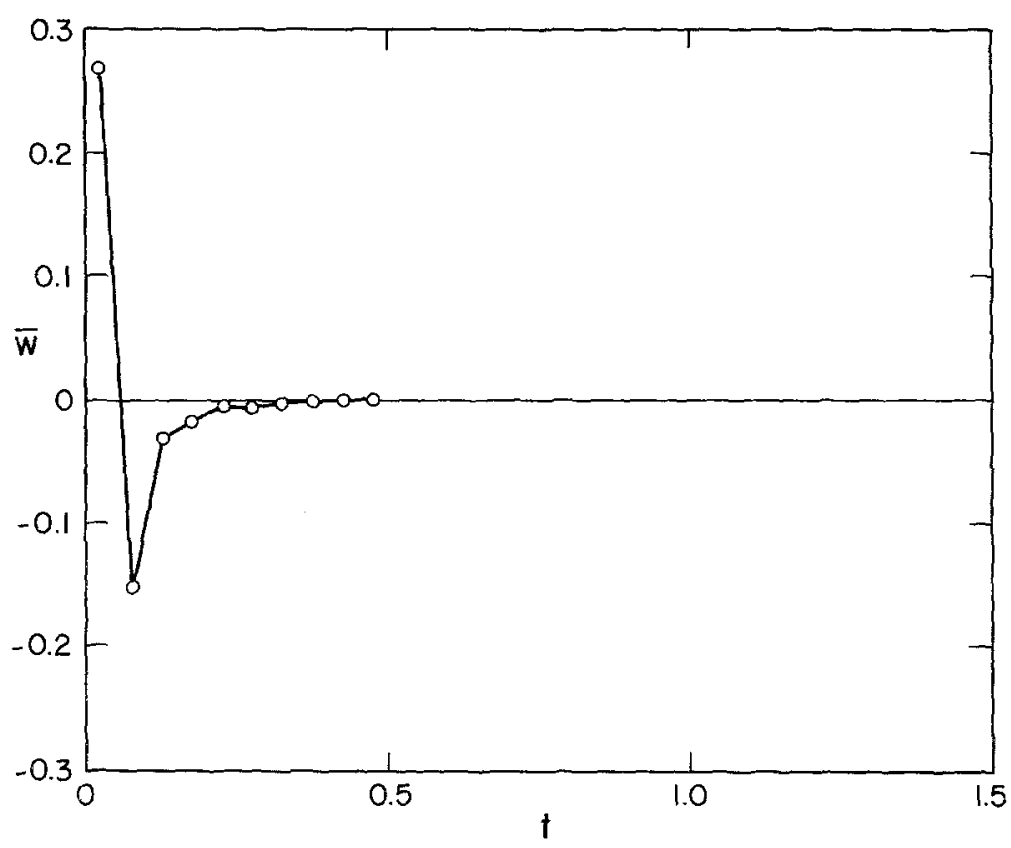

FIG. 4(a)

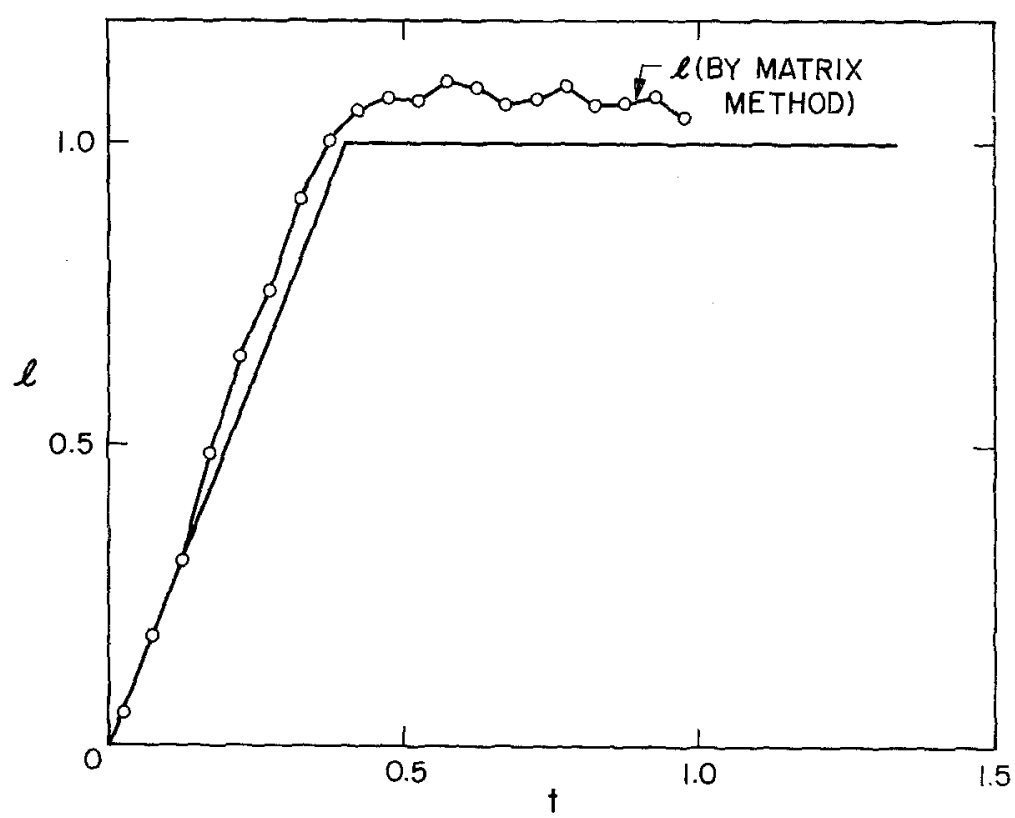

Fig. (b)

FIG. 4(a). Deconvolution function for the consolidation problem, $\bar{w}(t)$. (b). Calculated system input for the consolidation problem by the matrix technique, $l(t)$. 
Example 2. The same consolidation problem as used in Example 1 will be solved by this matrix method. For $w(t)$ as given in equation (11), the function $\bar{w}(t)$ corresponding to the deconvolution matrix $[\bar{W}]$ is obtained for $\Delta t=0.05$ as shown in Figure 4 a. Using this $[\bar{W}]$ in equation (19), the loading function $l(t)$ is obtained as shown in Figure 4b. Again, a good reproduction of the ramp loading is obtained.

Example 3. The next example concerns the scanning of a picture by a television vidicon camera. The scanning beam has a finite width and the camera therefore picks up signals from points adjacent to the one which is being scanned. This produces a reduced resolution in the picture, as shown in the pictures of the moon transmitted by Ranger and the Surveyor spacecraft.

A method has been used by Nathan (1966) to improve the pictures by allowing for the camera scanning function. Suppose a one-dimensional scene in $x$ with brightness $A(x)$, Figure 5, an example given by Nathan (1966), is to be scanned

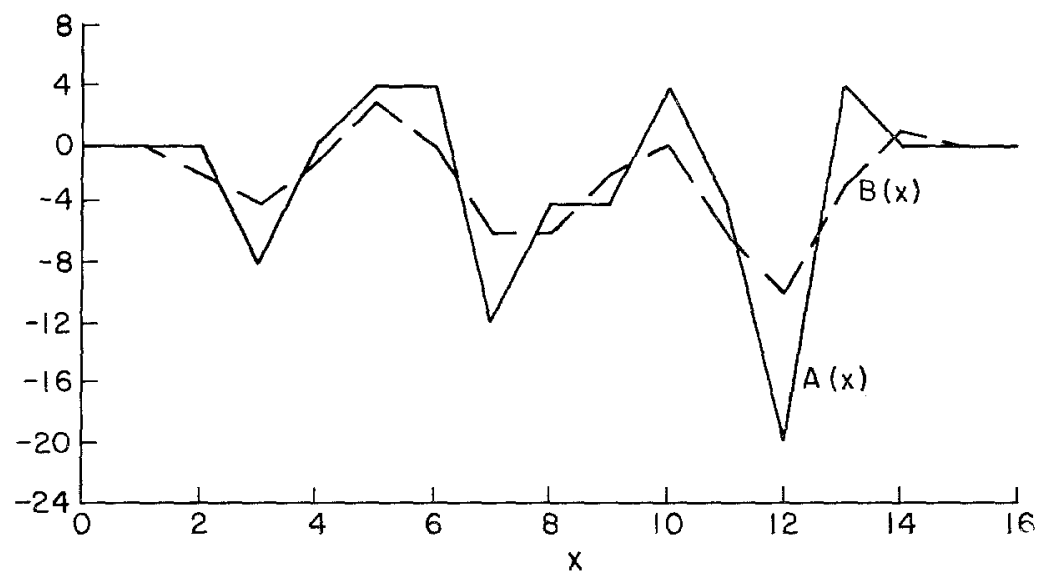

Fig. 5. Actual brightness, $A(x)$, and transmitted brightness $B(x)$ in Nathan's problem.

by a beam with a response function $S(x)$, where $S$ is as shown in Figure 6a. The transmitted brightness $B(x)$ is a convolution of $A$ and $S$.

$$
B_{i}=B\left(x_{i}\right)=\sum_{j=-2}^{2} A_{i+j} S_{j}, \quad i=1, \cdots 15,
$$

where $A_{i}=A\left(x_{i}\right)$, and $S_{i}=S\left(x_{i}\right)$. When written in full, this gives a set of linear algebraic equations similar to those in equation (17). From equation (22), $B(x)$ is obtained as shown in Figure 5. In this problem, $A$ is the input, $S$ the transfer or system response function and $B$ the output. Given $B$ and $S$, it is possible, by means of the deconvolution techniques developed above, to obtain $A$. Although the underlying theoretical principles were not discussed in his work, Nathan (1966) employed a Fourier transform technique similar to the one described above, but since the data on both $B$ and $S$ are digital in nature, it is doubtful whether the transform method should have been used. In Nathan's work a lower enhancement limit has to be imposed because the reciprocal of the transform has to be taken (similar to equation (6a)). This limit reduces the accuracy of his method and, as a result, only an approximate reproduction $R$ of $A$ is obtained as shown in Figure 7 . 
It is possible to use the deconvolution matrix method in problems like this where the data are digitized. With the transfer function $S$ shown in Figure (6)a, its inverse $\bar{S}$ can be obtained by means of equation (20) and this is shown in Figure (6)b. The original picture can now be obtained by convolving $\bar{S}$ with $B$, and it is found to be exactly the same as $A$ shown in Figure 5.

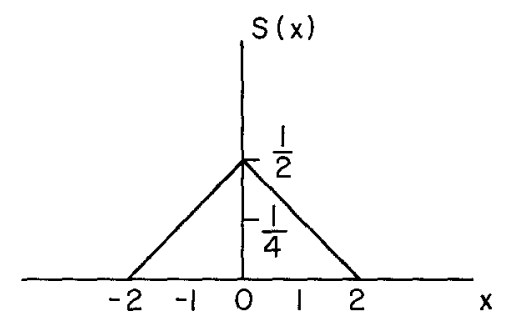

(a)

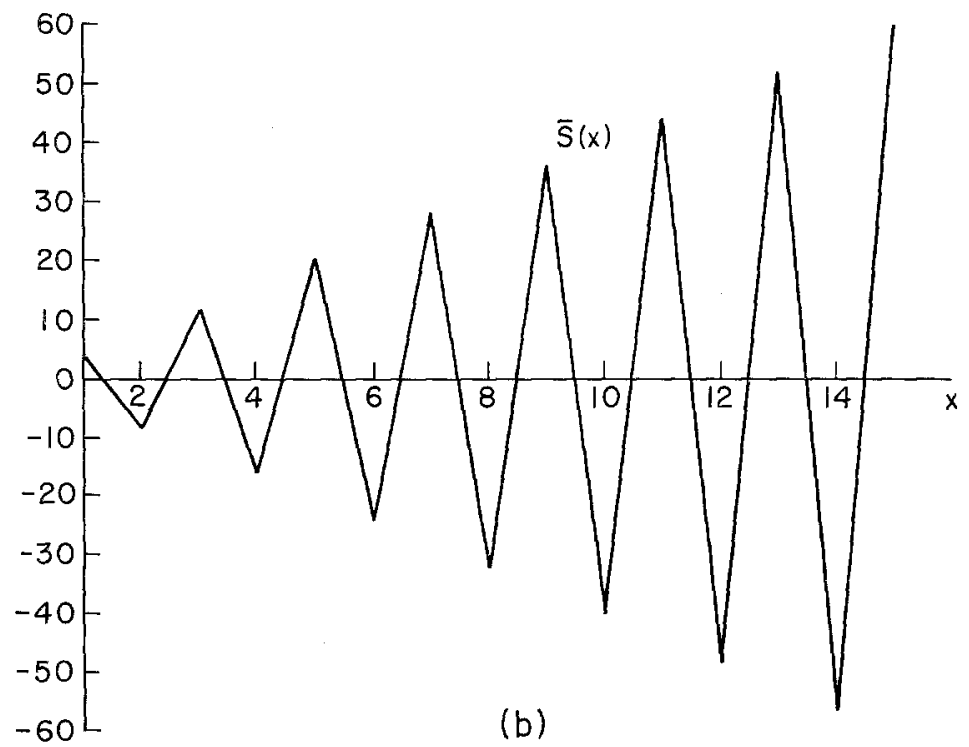

Fra. 6. System (Camera) function $S(x)$ and the deconvolution function $\bar{S}(x)$ for Nathan's problem.

In calculating the deconvolution matrix $[\bar{W}]$ by means of equation $(20)$, the number $w_{1}$ occurs repeatedly in the denominator of the $\bar{w}_{i}$ terms. For instance, $\bar{w}_{1}=$ $1 / w_{1}, \bar{w}_{2}=-w_{2} / w_{1}$ and so on. If the order $n$ of the problem is large, it is expected that, if $w_{1}$ is small, the numbers $\bar{w}_{i}$ become large as $i$ increases and oscillate at the same time, as shown in Figure 6b. However, if $w_{1}$ is large compared with other values of $w_{i}$, then $\bar{w}_{i}$ will not be divergent as $i$ increases. This is the case in the consolidation problem in Example 1. Even in such cases, if the order of the problem is large, the deconvolution matrix will not be accurate due to round-off errors. The calcula- 
tion of this matrix is actually equivalent to the inversion of the original convolution matrix $[W]$ but because of the peculiar property of $[W]$, the inversion can be accomplished much more readily than that of ordinary matrices. However, the problems encountered in ordinary matrix inversion are expected to exist in this case also. Therefore, the deconvolution matrix method may not be an efficient way of handling large problems.

One such large problem concerns the response of a mass-spring-damping system subjected to a random excitation. The behavior of the system can be described by the linear differential equation (Greenwood, 1965)

$$
m \ddot{x}+c \dot{x}+k x=l(t),
$$

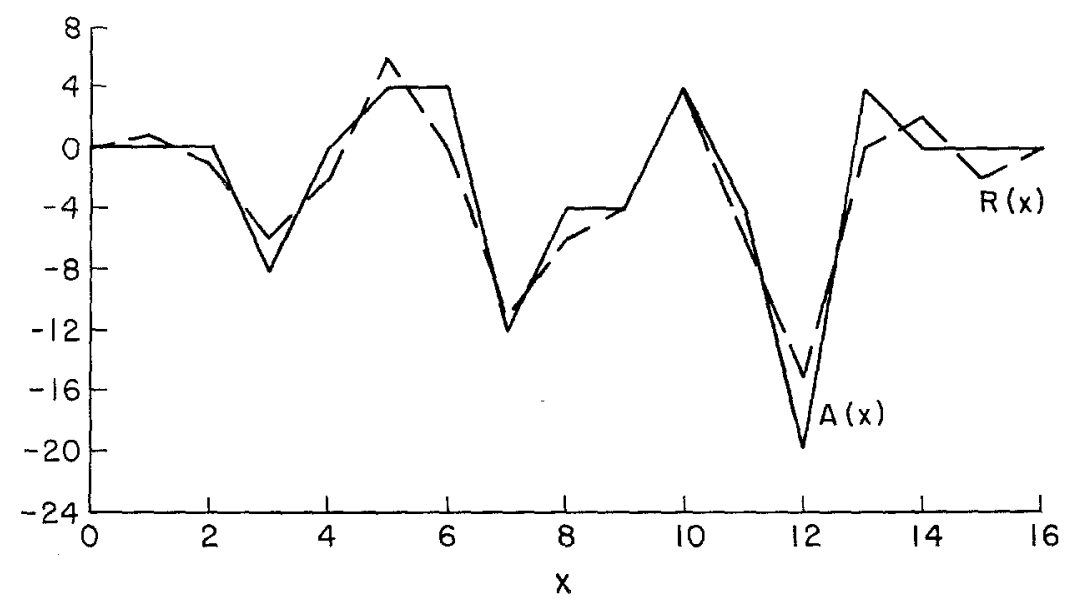

Fia. 7. Nathan's "enhanced" brightness, $R(x)$, compared to the actual brightness, $A(x)$.

or

$$
\ddot{x}+2 \zeta w_{n} \dot{x}+w_{n}^{2}=\frac{l(t)}{m} .
$$

If $l(t)$ and $x(t)$ are considered as the input and the output respectively, of this linear system, then the transfer function $w(t)$ is given by the solution of equation (23) when $l(t)$ is replaced by $\delta(t)$. Depending on the value of the damping $\zeta, w(t)$ is given by the following expressions.

For $\zeta=1.0$ (critically damped)

$$
w(t)=\frac{t}{m} e^{-\omega_{n} t} .
$$

For $\zeta<1.0$ (underdamped),

$$
w(t)=\frac{\omega_{n} e^{-\zeta \omega_{n} t}}{k \sqrt{1-\zeta^{2}}} \sin \omega_{n} \sqrt{1-\zeta^{2}} t .
$$


Equation (23) has wide applications. For example, it is the governing equation for most seismic recording instruments. It is, therefore, possible to use the deconvolution method to reproduce the ground motion $l$ from the seismograph record $x$ and the system function $w$.

Another example where equation (24) is applicable is the vibration of structures when subjected to shaking by earthquakes or machines. Between two points of the structure where the vibration can be measured, there is a transfer function which is characteristic of the structure, provided that there is no input to the structure between these points. In this case, there is some interest as to how this transfer function varies between different points in the structure, and it is also interesting to study the behavior of the structure when subjected to different earthquakes.

In order for the deconvolution methods developed here to work satisfactorily in practical situations, for example, deconvolving an earthquake record, it is necessary that the initial portions of the ground motion to be recorded also. In most earthquake recording instruments, the recording mechanism is triggered only by a certain threshold motion. Such records without the initial portions are useless as far as deconvolution is concerned, since, as shown above, deconvolution has to begin at the onset of the excitation. Another problem arises, for example, in the case where the transfer function is required between two locations in a structure subjected to earthquake motion; records taken by instruments situated at different locations are seldom correlated with respect to time and it is important that the relative time scale in both records (the input and the output) be known for the deconvolution.

In an investigation of these points, some calculations have been carried out for the linear mass-spring-damping system as described by equation 23, using as the input the artificial earthquake generated by Housner and Jennings (1964). The input was first convolved with the known system function to provide the output of the system. Next, the known input and the calculated output were deconvolved by the matrix method in an attempt to calculate the known system function. If the zeros on the time scales of each record were aligned exactly, it was found that the system function could be recovered almost exactly up to reasonably large times. However, by introducing a slight shift in the relative time scales of these records, the calculated system function began to deviate from the known function after a short time and diverged rapidly. This is caused in part by the small value of the first digitized reading in both the input and output, since, as pointed out earlier, this number appears in the denominator repeatedly. On the other hand, in example 2 on consolidation, the first number in the system function is not small and there the deconvolution method worked very well.

It appears that the deconvolution methods are of value in calculations for linear systems. However, where the size of the problem is large, refinement in the numerical technique is necessary to achieve the required accuracy.

It appears that the use of deconvolution may reduce the need for designing and utilizing a measuring instrument with an optimum system function for the process being measured. Alternatively, the output of an instrument responding to an input for which it is not designed may be used with these techniques to give better information on the real input. 


\section{ACKNOWLEDGMENT}

The work described in this paper was supported in part by a NSF Grant $1197 \mathrm{X}$ for research in earthquake engineering in the California Institute of Technology.

\section{References}

Bellmail, E. R., R. E. Kalaba and J. Lockett (1966). Numerical Inversion of the Laplace Transform, Elsevier, New York.

Beck, J. S. and A. Rescigno (1964). Determination of Precursor Order and Particular Weighting Functions from Kinetic Data, $J$. Theoret. Biol. 6, 1-12.

Carslaw, H. S. and J. C. Jaeger (1959). Conduction of Heat in Solids, Oxford University Press, New York.

Courant, R. and D. Hilbert (1953). Methods of Mathematical Physics, Interscience, New York. Fodor, G. (1965). Laplace Transforms in Engineering, Akadémiai Kiadó, Budapest.

Greenwood, D. T. (1965). Principles of Dynamics, Prentice-Hall, New Jersey.

Housner, G. W. and P. C. Jennings (1964). Generation of Artificial Earthquakes, J. Eng. Mech. Div., ASCE, 90, EM1, 113-150.

Nathan, R. (1966). Digital Video-Data Handling, Tech. Rpt. No. 32-877, Jet Propulsion Laboratory, California Institute of Technology.

Schapery, R. A. (1962). Approximate Methods of Transform Inversion for Viscoelastic Analysis, Proc. H th $_{\text {t }}$ U. S. Cong. of Appl. Mech. 2, 1075.

Schiffman, R. F. (1958). The Consolidation of Soil Under Time-Dependent Loading and Varying Permeability, Rensselaer Polytechnic Institute, Troy, New York.

Scott, R. F. (1963). Principles of Soil Mechanies, Addison-Wesley, Reading, Mass.

Triconi, F. G. (1957). Integral Equations, Interscience, New York.

Ter Haar, D. (1951). An Easy Approximation Method of Determining the Reaction Spectrum of a Viscoelastic Material, J. Pol. Sci. 6, 247.

UniversitTy of CoLorado

Boulder, Colorado

California Institute of Teghnology

Pasadena, Calitgornita

Manuscript received May 19, 1967. 